\title{
THE ABC TRANSPORTERS OF HUMAN PHYSIOLOGY AND DISEASE
}

Genetics and Biochemistry of ATP Binding Cassette Transporters 
This page intentionally left blank 


\section{THE ABC TRANSPORTERS OF HUMAN PHYSIOLOGY AND DISEASE}

Genetics and Biochemistry of ATP Binding Cassette Transporters

\section{Edited by \\ Kenneth J Linton \\ Queen Mary University of London, UK \\ I Barry Holland \\ Université Paris-Sud, France}




\section{Published by}

World Scientific Publishing Co. Pte. Ltd.

5 Toh Tuck Link, Singapore 596224

USA office: 27 Warren Street, Suite 401-402, Hackensack, NJ 07601

UK office: 57 Shelton Street, Covent Garden, London WC2H 9HE

\section{British Library Cataloguing-in-Publication Data}

A catalogue record for this book is available from the British Library.

\section{THE ABC TRANSPORTERS OF HUMAN PHYSIOLOGY AND DISEASE Genetics and Biochemistry of ATP Binding Cassette Transporters}

Copyright (C) 2011 by World Scientific Publishing Co. Pte. Ltd.

All rights reserved. This book, or parts thereof, may not be reproduced in any form or by any means, electronic or mechanical, including photocopying, recording or any information storage and retrieval system now known or to be invented, without written permission from the Publisher.

The front cover shows a cartoon based on the crystal structure of a related drug efflux ABC transporter (Sav1866) from Staphylococcus aureus. The relevance of this structure for our understanding of how human $\mathrm{ABC}$ transporters work is described in Chapter 1. The back cover shows an unrooted phylogenetic tree of the human $\mathrm{ABC}$ transporter family.

All 48 full length proteins were aligned in Clustal W and phylogenetic analysis was performed in Seaview.

For photocopying of material in this volume, please pay a copying fee through the Copyright Clearance Center, Inc., 222 Rosewood Drive, Danvers, MA 01923, USA. In this case permission to photocopy is not required from the publisher.

ISBN-13 978-981-4280-06-8

ISBN-10 981-4280-06-2

Typeset by Stallion Press

Email: enquiries@stallionpress.com

Printed in Singapore. 


\section{Contents}

Contributors $\quad$ xiii

Preface xxiii

Chapter 1 General Introduction, Structure 1 and Likely Mechanism of Action of ABC Transport Proteins

Kenneth J. Linton, Joseph K. Zolnerciks

and Lutz Schmitt

1. Introduction 1

2. The Human Complement of ABC Transporters 2

3. The Core Architecture of an ABC Transporter 3 Comprises Two TMDs and Two NBDs

4. Structure Analysis and Its Relevance to Molecular $\quad 7$ Mechanisms

5. Conformational Changes During the Transport Cycle 21

6. The ATP Switch Hypothesis 23

7. Future Perspectives 25

References $\quad 26$

Chapter 2 ABCA1-Mediated HDL Formation: 29 Mechanism and Regulation

Kohjiro Nagao, Masako Hozoji-Inada, Yuya Azuma and Kazumitsu Ueda

1. ABCA1 and HDL Formation

2. Structural Feature of ABCA1 30 
3. Mechanism of apoA-I-Dependent Cholesterol Secretion by ABCA1

4. Membrane Meso-Domains and ABCA1 Functions 33

5. SM Content and Functions of ABCA1 34

6. Substrate Transport Specificity 35

7. ApoA-I and Sodium Taurocholate, Lipid Acceptors for 36 ABCA1 and ABCB4

8. Four-Step Model for HDL Formation 37

9. Endocytosis and Recycling of ABCA1 38

10. Degradation of ABCA1 40

11. Regulation of ABCA1 by Interacting Proteins 41

12. Perspectives for the Future 44

Acknowledgments $\quad 45$

References $\quad 45$

Chapter 3 Bile Formation and the Enterohepatic 53 Circulation

Peter H. Dixon and Catherine Williamson

1. The Enterohepatic Circulation

2. ABC Transporters and Bile Formation - The Central Role of ABCB4 and ABCB11

3. Perspectives for the Future

References
Chapter 4 ABCB1 and ABCG2: Deciphering the Role of Human Efflux Proteins in Cellular and Tissue Permeability Marcos M. Pires, Dana M. Emmert, Jean A. Chmielewski and Christine A. Hrycyna

1. Introduction

2. Structural Studies of ABCB1 and ABCG2 89

3. Physiological Roles of ABCB1 and ABCG2 91

4. Drug Bioavailability 
5. Disease States Related to ABCB1 and ABCG2 104

6. Neurological Disease 106

7. Viral Disease 110

8. Multidrug Resistance in Cancer 111

9. Inhibition of ABCB1 and ABCG2 Transport 115 Activity in the Cancer Clinic

References

Chapter 5 ABCA4 and Its Role in the Visual Cycle and Inherited Retinal Degenerative Diseases

Robert S. Molday and Laurie L. Molday

1. Introduction

2. Overview of the Function of ABCA4 in the Photoreceptor Cells and the Mechanism Responsible for Stargardt Macular Degeneration

3. Localization of ABCA4 in Outer Segments of Photoreceptor Cells

4. Structural Features of ABCA4

5. Evidence for the Role of ABCA4 as a

N-Retinylidene-Phosphatidylethanolamine (N-Ret-PE) Transporter

6. Proposed Role of ABCA4 in the Clearance of N-ret-PE as Part of the Visual Cycle

7. Direction of Substrate Transport by ABCA4 - An Unresolved Issue

8. Inherited Retinal Degenerative Diseases Associated with Mutations in ABCA4

9. Accumulation of Toxic Retinal Compounds is Responsible for Stargardt Disease

10. Gene and Drug Based Therapeutic Treatments

11. Concluding Remarks 153

Acknowledgments $\quad 154$

References 
Chapter 6 The Role of the Antigen Translocation 163 Machinery TAP in Adaptive Immunity Daphne Nikles and Robert Tampé

1. Introduction

2. Organization and Function of the PLC 165

3. Structure and Function of TAP 168

4. Viral Immune Evasion Strategies Block 170 Peptide-loading

5. The Bare Lymphocyte Syndrome (BLS)

6. Tumor Escape Mechanisms Target TAP 174

7. Conclusions and Outlook 174

Acknowledgments 175

References

Chapter 7 The ABCs of Mitochondrial

181 Metabolic Disorders

Yu Fukuda, Dagny Ulrich, John Lynch and John D. Schuetz

1. Introduction

2. $\mathrm{ABCB} 6$

3. $\mathrm{ABCB} 7$

4. $\mathrm{ABCB} 8$

5. $\mathrm{ABCB} 10$

6. Conclusions and Perspectives 204

Acknowledgments 205

References

Chapter 8 The Role of ABCC Family Members in the Disposition of Endogenous Compounds and Drugs

Jörg König, Martin F. Fromm

and Hartmut Glaeser

1. MRP1 (Gene Symbol ABCC1)

2. $\quad$ MRP2 (Gene Symbol $A B C C 2)$

3. $\quad$ MRP3 (Gene Symbol ABCC3) 
4. MRP4 and MRP5 (Gene Symbols ABCC4 and ABCC5) 224

5. $\quad$ MRP6 (Gene Symbol ABCC6) 231

6. Summary and Perspective 233

References 234

Chapter 9 ABC Transporter Mutations in

247 Heritable Skin Diseases: The $A B C C 6$ and $A B C A 12$ Genes

Qiaoli Li, Qiujie Jiang and Jouni Uitto

1. Introduction 247

2. The Paradigms of Pseudoxanthoma Elasticum and 250 Harlequin Ichthyosis

3. Clinical Implications of Mutation Analysis in 263 PXE and HI

Acknowledgments 264

References $\quad 265$

Chapter 10 CFTR: Understanding the Cause 269 and Influencing the Outcome of a Major Genetic Disease

Tamás Hegedüs, Martina Gentzsch and John R. Riordan

1. CFTR Function

2. Structural Properties of CFTR 274

3. CFTR Synthesis and Assembly 281

4. Intracellular Trafficking of CFTR 288

5. Overcoming Cystic Fibrosis Causing Mutations 292 References

Chapter 11 ABCC8: Physiology and Pathology

David Ortiz, Yumiko Nakamura, Hiroshi Hashiguchi, Joseph Bryan and Lydia Aguilar-Bryan

1. Introduction

2. Channel Topology and Assembly 
3. Dual Regulation of $\mathrm{K}_{\mathrm{ATP}}$ by Nucleotides 313

4. Pharmacology 316

5. $\mathrm{K}_{\mathrm{ATP}}$ Channels in the Pancreatic Islets 320 of Langerhans

6. $\mathrm{K}_{\text {ATP }}$ Channels in Insulin-Secreting $\beta$-Cells 321

7. $\mathrm{K}_{\mathrm{ATP}}$ in Glucagon-Secreting $\alpha$-Cells 323

8. $\mathrm{K}_{\mathrm{ATP}}$ in Somatostatin Secreting $\delta$-Cells 327

9. Summary of Intra-Islet Regulation 328

10. Disease States $\quad 330$

11. Hyperinsulinemic Hypoglycemia of Infancy 330

12. Neonatal Diabetes Mellitus 333

13. Diagnosis and Implications for Therapy 334 References 335

Chapter 12 The ABCD Subfamily: Peroxisomal Transporters in Health and Disease Aurora Pujol and Patrick Aubourg

1. Introduction

2. X-Linked Adrenoleukodystrophy (X-ALD): A Complex Disease Due to Loss of Function of ABCD1 Gene

3. The ABCs of Peroxisomes

4. Fatty Acid Transport Across the Peroxisomal Membrane: The Role of ABCD-Family Transporters

5. The ABCDs of Baker's Yeast

6. The ABCDs of Mammals 358

7. The Function of ABCD1 (ALDP) Transporter 359

8. Do Mammalian Peroxisomal ABCD Half-Transporter 361 Act as Homo- or Heterodimers?

9. ABCD2, ABCD3 and ABCD4 Transporters and Their 363 Overlapping Roles in Fatty Acid Metabolism

10. Inducible Expression of the Mammalian ABCD

Proteins and Relevance for X-ALD

11. Conclusion

Acknowledgments $\quad 370$

References 
Chapter 13 ABCG5/8 and Its Role in Sterol

Homeostasis

Ronald P. J. Oude Elferink

and Coen C. Paulusma

1. Introduction

375

2. The ABC Transporter Couple ABCG5/ABCG8 376

3. Sitosterolemia 378

4. Intestinal Sterol Absorption and Excretion 380

5. Biliary Cholesterol Secretion 382

6. The Role of Bile in Cholesterol Homeostasis 387

7. ABCG5/8 and Gallstones 388

$\begin{array}{ll}\text { References } & 391\end{array}$

Chapter 14 Hot Topics, Recent Advances 397 and Novel Approaches for Clinical Intervention

Irwin M. Arias, Saskia W. C. van Mil, Heidrun Potschka, Alan S. Verkman, Uta Griesenbach, Eric W. F. W. Alton, Balazs Sarkadi and Gergely Szakacs

\footnotetext{
Introduction

1. The ABC of Hepatobiliary ABC-Transporter Regulation by Nuclear Receptors

2. Targeting Regulation of ABC Transporters 405

3. Small-Molecule Corrector Therapy for Cystic Fibrosis 411

4. Gene Therapy for Cystic Fibrosis 416

5. The Role of ABC Multidrug Transporters 424 in Normal and Cancer Stem Cells

References
}

397

Index 
This page intentionally left blank 


\section{Contributors}

\section{Lydia Aguilar-Bryan, MD, PhD}

Principal Scientist

Pacific Northwest Diabetes Research Institute

720 Broadway

Seattle, WA 98122, USA

\section{Eric W. F. W. Alton, MD, FRCP, FmedSci}

Department of Gene Therapy

Imperial College London

Manresa Road

London SW3 6LR, UK

\section{Irwin M. Arias, MD}

Senior Scientist

Head Unit on Cellular Polarity

Cell Biology and Metabolism Program

National Institute of Child Health and Human Development

National Institutes of Health

Bethesda, MD 20892, USA

\section{Patrick Aubourg, MD}

Faculty of Pharmaceutical and Biological Sciences

Inserm U745 and University Paris Descartes

4 avenue de l'Observatoire

75279, Paris cedex 06, France 


\section{Yuya Azuma, PhD}

Laboratory of Cellular Biochemistry

Division of Applied Life Sciences

Graduate School of Agriculture, Kyoto University

Kitasirakawa, Sakyo-ku

Kyoto 606-8502, Japan

Joseph Bryan, PhD

Principal Scientist

Pacific Northwest Diabetes Research Institute

720 Broadway

Seattle, WA 98122, USA

\section{Jean A. Chmielewski, PhD}

Department of Chemistry

Purdue University

560 Oval Dr.

West Lafayette, IN 47907-2084, USA

\section{Peter H. Dixon, PhD, BSc}

Maternal and Fetal Disease Group

Institute of Reproductive and Developmental Biology

Department of Surgery and Cancer

Imperial College Hammersmith Campus

Du Cane Road

London W12 0NN, UK

\section{Dana M. Emmert, PhD}

Department of Chemistry

Purdue University

560 Oval Dr.

West Lafayette, IN 47907-2084, USA 


\section{Martin F. Fromm, MD}

Institute of Experimental and

Clinical Pharmacology and Toxicology

Department of Clinical Pharmacology and Clinical Toxicology

Friedrich-Alexander Universität Erlangen-Nürnberg

Fahrstrasse 17

91054 Erlangen, Germany

\section{Yu Fukuda, PhD}

Department of Pharmaceutical Sciences

St. Jude Children's Research Hospital

262 Danny Thomas Place - MS 313

Memphis, TN 38105, USA

\section{Martina Gentzsch, PhD}

Cystic Fibrosis Research Center and

Department of Cell and Developmental Biology

University of North Carolina, Chapel Hill, North Carolina

6103 Thurston-Bowles Bldg., CB\# 7248

Chapel Hill, NC 27599-7248, USA

\section{Hartmut Glaeser, PhD}

Institute of Experimental and

Clinical Pharmacology and Toxicology

Department of Clinical Pharmacology and Clinical Toxicology Friedrich-Alexander Universität Erlangen-Nürnberg

Fahrstrasse 17

91054 Erlangen, Germany

\section{Uta Griesenbach, PhD}

Department of Gene Therapy

Imperial College London

Manresa Road

London SW3 6LR, UK 


\section{Hiroshi Hashiguchi, MD, PhD}

Postdoctoral fellow

Pacific Northwest Diabetes Research Institute

720 Broadway

Seattle, WA 98122, USA

\section{Tamás Hegedüs, PhD}

Cystic Fibrosis Research Center and

Department of Biochemistry and Biophysics

University of North Carolina, Chapel Hill, North Carolina

6103 Thurston-Bowles Bldg., CB\# 7248

Chapel Hill, NC 27599-7248, USA

\section{Masako Hozoji-Inada, PhD}

Institute for Integrated Cell-Material Sciences (iCeMS)

Kyoto University

Kitasirakawa, Sakyo-ku

Kyoto 606-8502, Japan

\section{Christine A. Hrycyna, PhD}

Department of Chemistry

Purdue University

560 Oval Dr.

West Lafayette, IN 47907-2084, USA

\section{Qiujie Jiang, PhD}

Assistant Professor

Department of Dermatology and Cutaneous Biology

Jefferson Medical College

Thomas Jefferson University

233 S. $10^{\text {th }}$ Street, BLSB 430

Philadelphia, PA 19107, USA 


\section{Jörg König, PhD}

Institute of Experimental and

Clinical Pharmacology and Toxicology

Department of Clinical Pharmacology and Clinical Toxicology

Friedrich-Alexander Universität Erlangen-Nürnberg

Fahrstrasse 17

91054 Erlangen, Germany

\section{Qiaoli Li, PhD}

Assistant Professor

Department of Dermatology and Cutaneous Biology

Jefferson Medical College

Thomas Jefferson University

233 S. $10^{\text {th }}$ Street, BLSB 430

Philadelphia, PA 19107, USA

\section{Kenneth J. Linton, PhD}

Blizard Institute of Cell and Molecular Science

Barts and The London School of Medicine and Dentistry

Queen Mary, University of London

4 Newark Street, Whitechapel

London E1 2AT, UK

\section{John Lynch, PhD}

Department of Pharmaceutical Sciences

St. Jude Children's Research Hospital

262 Danny Thomas Place - MS 313

Memphis, TN 38105, USA

\section{Laurie L. Molday, BSc}

Department of Biochemistry and Molecular Biology

Centre for Macular Research

University of British Columbia

2350 Health Sciences Centre

Vancouver, B.C. V6T 1Z3, Canada 


\section{Robert S. Molday, PhD, FRSC}

Department of Biochemistry and Molecular Biology

Centre for Macular Research

University of British Columbia

2350 Health Sciences Centre

Vancouver, B.C. V6T 1Z3, Canada

\section{Kohjiro Nagao, PhD}

Institute for Integrated Cell-Material Sciences (iCeMS)

Kyoto University

Kitasirakawa, Sakyo-ku

Kyoto 606-8502, Japan

\section{Yumiko Nakamura, MD, PhD}

Postdoctoral Fellow

Pacific Northwest Diabetes Research Institute

720 Broadway

Seattle, WA 98122, USA

\section{Daphne Nikles, PhD}

Institute of Biochemistry

Biocenter, Goethe-University Frankfurt

Max-von-Laue Str. 9

D-60438 Frankfurt a.M., Germany

\section{David Ortiz}

Graduate Student in the Program of Structural and

Computational Biology and Molecular Biophysics

at Baylor College of Medicine

Pacific Northwest Diabetes Research Institute

720 Broadway

Seattle, WA 98122 , USA 


\section{Ronald P. J. Oude Elferink, PhD}

Tytgat Institute for Liver and Intestinal Research

Academic Medical Center, S1-162

Meibergdreef 69-71

1105 BK Amsterdam, The Netherlands

\section{Coen C. Paulusma, PhD}

Tytgat Institute for Liver and Intestinal Research

Academic Medical Center, S1-162

Meibergdreef 69-71

1105 BK Amsterdam, The Netherlands

\section{Marcos M. Pires, PhD}

Department of Chemistry

Purdue University

560 Oval Dr.

West Lafayette, IN 47907-2084, USA

\section{Heidrun Potschka, DVM}

Institute of Pharmacology, Toxicology, and Pharmacy

Ludwig-Maximilians-University Munich

Koeniginstr. 16

80539 Munich, Germany

\section{Aurora Pujol, MD, PhD}

Institut d'Investigació Biomèdica de Bellvitge

Gran Via de l'Hospitalet, 199

08907 L'Hospitalet de Llobregat

Barcelona, Spain

\section{John R. Riordan, PhD}

Cystic Fibrosis Research Center and

Department of Biochemistry and Biophysics

University of North Carolina, Chapel Hill, North Carolina 6103 Thurston-Bowles Bldg., CB\# 7248

Chapel Hill, NC 27599-7248, USA 
Balazs Sarkadi, MD, PhD, DSc

Membrane Research Group

Hungarian Academy of Sciences

Dioszegi 64, 1113 Budapest, Hungary

\author{
Lutz Schmitt, PhD \\ Institute of Biochemistry \\ Heinrich Heine University Düsseldorf \\ Universitätsstr. 1 \\ 40225 Düsseldorf, Germany
}

\title{
John D. Schuetz, PhD
}

Member, Vice Chair

Department of Pharmaceutical Sciences

St. Jude Children's Research Hospital

262 Danny Thomas Place — MS 313

Memphis, TN 38105, USA

\section{Gergely Szakacs, MD, PhD}

Institute of Enzymology

Hungarian Academy of Sciences

Karolina 29, 1113 Budapest, Hungary

\section{Robert Tampé, PhD}

Institute of Biochemistry

Biocenter, Goethe-University Frankfurt

Max-von-Laue Str. 9

D-60438 Frankfurt a.M., Germany

\section{Kazumitsu Ueda, PhD}

Institute for Integrated Cell-Material Sciences (iCeMS) and

Laboratory of Cellular Biochemistry

Division of Applied Life Sciences

Graduate School of Agriculture, Kyoto University

Kitasirakawa, Sakyo-ku

Kyoto 606-8502, Japan 


\section{Jouni Uitto, MD, PhD}

Professor and Chair

Department of Dermatology and Cutaneous Biology

Jefferson Medical College and

Director of Jefferson Institute of Molecular Medicine Thomas Jefferson University 233 S. $10^{\text {th }}$ Street, BLSB 450 Philadelphia, PA 19107, USA

\section{Dagny Ulrich, PhD}

Department of Pharmaceutical Sciences

St. Jude Children's Research Hospital

262 Danny Thomas Place - MS 313

Memphis, TN 38105, USA

\section{Saskia W. C. van Mil, PhD}

Department of Metabolic and Endocrine Diseases and

Netherland's Metabolomics Center

Universitair Medisch Centrum Utrecht

Room KC02.069.0, Lundlaan 6

3584 EA Utrecht, The Netherlands

\section{Alan S. Verkman, MD, PhD}

Department of Medicine and Physiology

University of California, San Francisco

1246 Health Sciences East Tower, Box-521

San Francisco, CA 94143, USA

\section{Catherine Williamson, MD, FRCP}

Professor of Obstetric Medicine

Institute of Reproductive and Developmental Biology

Department of Surgery and Cancer

Imperial College Hammersmith Campus

Du Cane Road

London W12 0NN, UK 


\section{Joseph K. Zolnerciks, PhD}

Blizard Institute of Cell and Molecular Science

Barts and The London School of Medicine and Dentistry Queen Mary, University of London

4 Newark Street, Whitechapel London E1 2AT, UK 


\section{Preface}

ATP binding cassette (ABC) proteins are versatile machines that convert chemical energy from the hydrolysis of ATP into mechanical energy. These domains can be used to drive diverse cellular processes such as chromatin organization, DNA repair and mRNA trafficking, but the vast majority power the flow of solutes across cellular membranes.

ATP binding cassette transport proteins are integral membrane proteins that catabolize ATP to drive the uptake (prokaryota only) or efflux (prokayota and eukaryota) of solutes across membranes. Their ubiquity and abundance (many different forms are found in all species) is indicative of their importance to the maintenance of a healthy cell, organ and organism with an impact both directly and indirectly on human health. Chloroquine resistance in Plasmodium sp., antibiotic efflux from bacterial producers and some pathogenic bacteria, and fungal-pathogen resistance in wheat, remind us that you do not have to stray far from a human centric view of the biosphere to see the influence of this superfamily of transporters.

This volume focuses specifically on the direct influence imparted by the complement of more than $40 \mathrm{ABC}$ transporters encoded by the human genome. Increasingly, studies from laboratory bench to clinical care reveal that human $\mathrm{ABC}$ transporters are crucial to both normal physiology and pathology. These include important roles, for example, in haem, lipid and fatty acid metabolism, bile flow, drug disposition and ion flux, which encompass nearly every organ, tissue and cell type in the body. 
Their malfunction or dysregulation is linked to more than 30 diseases and conditions, and it seems likely that more will be discovered as disease aetiologies, or the precise function of some of the transporters, are clarified.

This is a particularly exciting time for those in the $A B C$ transporter research field. The recent FEBS special meeting on ABC transporters in Innsbruck in 2010 attracted 300 delegates committed to the analysis of the structure, biochemistry and genetics of diverse $\mathrm{ABC}$ transporters from many species. The field is certainly broad but the seemingly highly conserved fundamentals of $\mathrm{ABC}$ transporter mode of action ensures that those of us who work in the realm of biomedical sciences can learn from the advances in distant disciplines; from the structure and function of bacterial transporters, to the role of plant transporters in lipid or wax metabolism. Although we now have a reasonable idea of the general principles driving the action of these transporters, we still lack details of crucial aspects of the mechanism, including the nature of endogenous allocrites (the transported "substrates"), how these proteins fold and how the expression of individual $\mathrm{ABC}$ transporter genes are regulated. With greater understanding of the basic science and knowledge of disease aetiology, this will afford the development of diagnostic and prognostic tools, and consequently more informed clinical intervention. Concerning the latter, several recent examples herald much promise: gene therapy for the neurodegenerative disease, X-linked adrenoleukodystrophy; the development and use of small molecules to switch on the chaperones needed to facilitate maturation and function of folding mutants of the cystic fibrosis transmembrane regulator. One of the most difficult challenges remains the design of specific drugs for clinical use that can successfully modulate the action of the multidrug efflux ABC transporters, without serious side effects. More distant objectives include drug therapy developed on personalized lines, and the targeting of new drugs to circumvent the action of the resident $\mathrm{ABC}$ transporters. This will allow penetration of specific barriers to treat, for example, brain tumors, epilepsy or psychotic 
disease. If such aims are to be achieved, this is certainly not the time to restrict research activity in this field. On the contrary we would very much encourage young scientists to apply their skills to the study of these fascinating proteins not least in the clinical environment. 


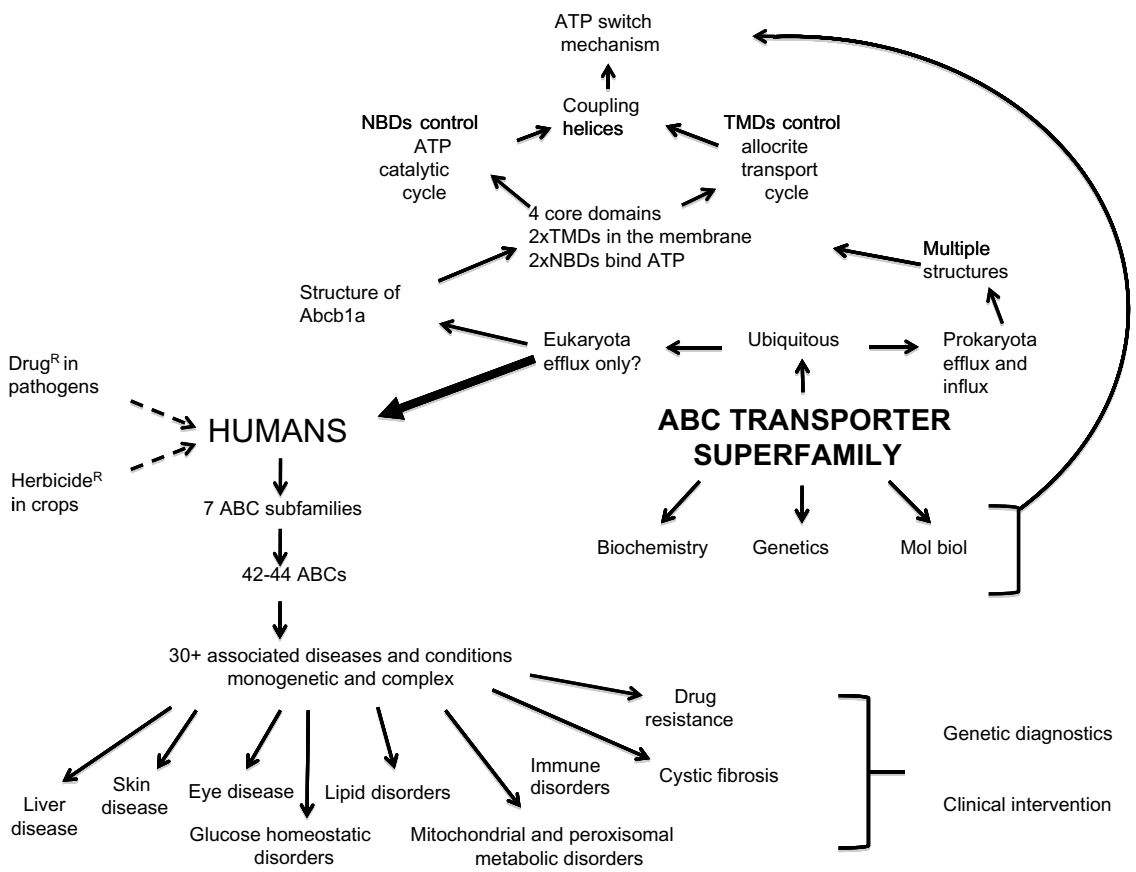

